1st Edition

\title{
Death, Grief and Loss in the Context of COVID-19
}

Edited By

\section{Panagiotis Pentaris}

Copyright Year 2021

ISBN 9780367647322

Published August 6, 2021 by Routledge

300 Pages 3 B/W Illustrations

\section{Death, Grief and Loss in the Context of COVID-19}

\section{What are VitalSource eBooks?}

Routledge \& CRC Press eBooks are available through VitalSource. The free VitalSource Bookshelf ${ }^{\circledR}$ application allows you to access to your eBooks whenever and wherever you choose.

- Mobile/eReaders - Download the Bookshelf mobile app at VitalSource.com or from the iTunes or Android store to access your eBooks from your mobile device or eReader.

- Offline Computer - Download Bookshelf software to your desktop so you can view your eBooks with or without Internet access.

- Learn More about VitalSource Bookshelf »

- Bookshelf Online Browser version support »

- System requirements for Bookshelf for PC, Mac, IOS and Android etc. »

Most VitalSource eBooks are available in a reflowable EPUB format which allows you to resize text to suit you and enables other accessibility features. Where the content of the eBook requires a specific layout, or contains maths or other special characters, the eBook will be available in PDF (PBK) format, which cannot be reflowed. For both formats the functionality available will depend on how you access the ebook (via Bookshelf Online in your browser or via the Bookshelf app on your PC or mobile device).

\section{Book Description}


This book provides detailed analysis of the manifold ways in which COVID-19 has influenced death, dying and bereavement.

Through three parts: Reconsidering Death and Grief in Covid-19; Institutional Care and Covid-19; and the Impact of COVID-19 in Context, the book explores COVID-19 as a reminder of our own and our communities' fragile existence, but also the driving force for discovering new ways of meaning-making, performing rites and rituals, and conceptualising death, grief and life. Contributors include scholars, researchers, policymakers and practitioners, accumulating in a multi-disciplinary, diverse and international set of ideas and perspectives that will help the reader examine closely how Covid-19 has invaded social life and (re)shaped trauma and loss.

It will be of interest to all scholars and students of death studies, biomedicine, and end of life care as well as those working in sociology, social work, medicine, social policy, cultural studies, anthropology, psychology, counselling and nursing more broadly.

\section{Table of Contents}

Introduction: Capturing the beginning of a long journey of loss, trauma and grief Panagiotis Pentaris

\section{PART 1: Reconsidering Death and Grief in Covid-19}

Chapter 1. Familiarity with death

Panagiotis Pentaris and Kate Woodthorpe

Chapter 2: Grief in the COVID-19 pandemic

Kenneth Doka

Chapter 3: Apocalypse now: COVID-19 and the crisis of meaning

Robert Neimeyer, Evgenia Milman and Sherman Lee

Chapter 4: Physically distant but socially connected: Streaming funerals, memorials and ritual design during COVID-19

Stacey Pitsillides and Jayne Wallace

Chapter 5: Social death in 2020: Covid-19, which lives matter and which deaths count? Jana Králová

PART 2: Institutional Care and Covid-19

Chapter 6: End-of-life decision-making in the context of a pandemic Natalie Pattison and Lucy Ryan

Chapter 7: NHS Values, Ritual, Religion, and Covid-19 Death

Douglas Davies

Chapter 8: Non-COVID-19 related dying and death during the pandemic

Wai Yee Chee, Samuel Wang, Winnie Teo, Melissa Fong, Andy Lee and Woon Chai Yong 
Chapter 9: Covid-19 and care home deaths and harms: A case study from the UK Alisoun Milne

Chapter 10: Impact of Covid-19 on mental health and associated losses

Manju Shahul-Hameed, John Foster, Gina Finnerty and Panagiotis Pentaris

Chapter 11: Assisted dying and Covid-19

Theo Boer and Kevin Yuill

PART 3: Impact of COVID-19 in Context

Chapter 12: Losing touch? Older people and COVID-19

Renske Claasje Visser

Chapter 13: Between cultural necrophilia and African American activism: life and loss in the age of COVID

Kami Fletcher and Tamara Waraschinski

Chapter 14: The biopolitics and stigma of the HIV and Covid-19 Pandemics

Jason Schaub

Chapter 15: Suicide in the context of the COVID-19 pandemic

Mohammed Mamun and Jannatul Mawa Misti

Chapter 16: Death and dying during the COVD-19 pandemic: The Indian context Apurva Kumar Pandya and Khyati Tripathi

\section{Editor(s)}

\section{Biography}

Panagiotis Pentaris is an Associate Professor of Social Work and Thanatology in the School of Human Sciences at the University of Greenwich, London, England, UK, where he is also a member of the Institute for Lifecourse Development, an internationally recognised Institute focusing on interdisciplinary research across the lifespan. Pentaris is a council member for the Association for the Study of Death and Society, and over the last ten years he has researched and published on death, dying, bereavement, culture and religion, social work, social policy and LGBTQIA+ issues.

Book Series

This book is included in the following series:

- Routledge Advances in Health and Social Policy 